Pacific Journal of Mathematic 


\section{ON THE TOWER THEOREM FOR FINITE GROUPS}

\section{Eugene Schenkman}

Wielandt [2] has given a very ingenious proof of the fact that the tower of automorphisms of a finite group without center ends after a finite number of steps. Using his work as a model a proof of a similar tower theorem for Lie algebras was given in [1]. This depends on the following three facts:

(a) If $A$ (with no center) is a member of the tower of derivation algebras of a Lie algebra $L$ then the centralizer of $L$ in $A$ is (0).

(b) If $L$ is a subinvariant Lie algebra of $A$ and if the centralizer of $L$ in $A$ is (0) then the centralizer of $L^{\omega}$ in $A$ is contained in $A$.

(c) If $L$ is subinvariant in $A$ then $L^{\omega}$ is normal in $A$.

In view of the much sharper estimate obtained in the theorem on Lie algebras it seemed to be of interest to attempt to improve on the results of Wielandt using the method of [1]. The group theory analogue of (a) is to be found in Wielandt's work. I shall prove here the analogue to (b) and then show by a counter-example that the method is not applicable to get the tower theorem even for solvable groups since the analogue to (c) does not hold for groups even under the additional hypothesis of (b).

Theorem. If $G$ is a subinvariant subgroup of the finite group $A$ and if the centralizer of $G$ in $A$ is the identity, then the centralizer of $G^{\omega}$ in $A$ is contained in $G^{\omega}$. It follows that if $N$ is normal in $G$ such that $G / N$ is nilpotent then $N \supset G^{\omega}$ and the centralizer of $N$ is contained in $N$.

Here

$$
G^{\omega}=\bigcap_{h=1}^{\infty} G^{k}
$$

where $G^{k}=\left[G^{k-1}, G\right]$ is the subgroup generated by commutators of the form $[h, g]=h g h^{-1} g^{-1}, h \in G^{k-1}, g \in G$.

The proof of the Theorem depends on two lemmas.

Lemma 1. If $G$ is a finite group then $G=G^{\omega} H$ where $H$ is a nilpotent subgroup of $G$.

Received July 19, 1954. This research was supported by the U.S. Air Force under contract number AF19 (600)-790 monitored by the Office of Scientific Research. 
LEMMA 2. Let $G$ be a group with center $E$; then the centralizer $W$ of $G^{\omega}$ in $G$ is contained in $G^{\omega}$. It follows that if $N$ is normal in $G$ such that $G / N$ is nilpotent then $N \supset G^{\omega}$ and the centralizer of $N$ is contained in $N$.

I shall also give an example to show that under the hypotheses of the Theorem $G^{\omega}$ need not be normal in $A$, even with the added restriction that $G$ be solvable.

Proof of Lemma 1. The proof is based on the fact that a group is nilpotent if and only if its $\Phi$-subgroup contains the commutator subgroup $G^{2}[3, \mathrm{p}$. 114]. If $G$ is nilpotent the theorem is trivially true since $G^{\omega}=E$ and $G=G E$. If $G$ is not nilpotent then the $\Phi$-subgroup does not contain $G^{2}$. Accordingly we can pick a minimal set of generators $g_{1}, \cdots, g_{k}$ of $G$ where at least one of the generators, $g_{k}$ for definiteness, is in $G^{2}$. Then $g_{1}, \cdots, g_{k-1}$ generate a proper subgroup $K$ of $G$; and $G=K G^{2}$. On the other hand $G / G^{\omega}$ is nilpotent and hence the $\Phi$-subgroup of $G / G^{\omega}$ contains the commutator subgroup of $G / G^{\omega}$. Accordingly $g_{k} G^{\omega}$ is not essential as a generator of $G / G^{\omega}$ and therefore $g_{1} G^{\omega}, \cdots, g_{k-1} G^{\omega}$ generate $G / G^{\omega}$. It follows that $G=G^{\omega} K$.

Now we proceed by induction on the order of the group. Since $K$ is a proper subgroup of $G$, its order is less than that of $G$ and we can assume that $K=K^{\omega} H$ where $H$ is a nilpotent group. Then

$$
G=G^{\omega} K=G^{\omega} K^{\omega} H=G^{\omega} H
$$

since $K^{\omega}$ is contained in $G^{\omega}$ and the lemma is proved.

Proof of Lemma 2. $G^{\omega}$ is normal in $G$ and hence so also is $W$. By Lemma $1, G=G^{\omega} H$ where $H$ is a nilpotent group. Let $G_{1}=W H$. Then $G_{1}$ is a group since $W$ is normal and $H$ is a group. Also $G_{1}=$ $G_{1}^{\omega} H_{1}$ where $H_{1}$ is nilpotent. But $G_{1}^{\omega}=(W H)^{\omega}$ is contained in $W$ since $H$ is nilpotent and $W$ is normal. This can be seen by showing inductively that $(W H)^{k} \subseteq W H^{k}$. For let $x$ and $y$ be elements of $W, h$ be in $H$, and $k$ be in $H^{k}$. Then if $x h$ is in $W H$ and if $y k$ is in $W H^{k}$

$$
[x h, y k]=\underline{x h y h^{-1}} h k h^{-1} x^{-1} h k^{-1} h^{-1} h k h^{-1} k^{-1} y^{-1}[h, k]^{-1}[h, k]
$$

which is an element of $W H^{k+1}$, since the four underlined expressions are in $W$ and $[h, k] \in H^{k+1}$.

Now if $W$ is contained in $G_{1}^{\omega}$ then $W \subseteq G^{\omega}$ since $G_{1}^{\omega} \subseteq G^{\omega}$. Hence the lemma is false only if $W \downarrow G_{1}^{\omega}$. We need only consider therefore if there is an element $w$ in $W, w$ not in $G_{1}^{\omega}$. We shall write $w=g h$ where $g$ is in $G_{1}^{\omega}$ and $h$ in $H_{1}$. Of course $h \neq e$ since then $w$ would be in $G_{1}^{\omega}$. It follows that $h=g^{-1} w$ is in $W$, since $W \supseteq G_{1}^{\omega}$ and therefore $H_{1} \cap W \neq$ $E$. But $H_{1} \cap W$ is normal in $H_{1}$ since $W$ is normal in $G$. Thus $H_{1} \cap W$ 
has intersection $P \neq E$ with the center of $H_{1}$. But this will imply that $P$ is in the center of $G$. For $G=G^{\omega} H=G^{\omega} H_{1}$ since $H \subseteq G_{1}^{\omega} H_{1}$; and $P$ is in the centralizer of $G^{\omega}$ and in the center of $H_{1}$. We have shown that if the centralizer of $G^{\omega}$ is not contained in $G^{\omega}$ then $G$ has center not equal to $E$ contradicting the hypothesis of the lemma.

Proof of the Theorem. By Lemma 2 we know that if $Z$ is the centralizer of $G^{\omega}$ in $A$ then $Z \cap G \subseteq G^{\omega}$ since otherwise $G$ would have a non-trivial center. Now if $Z$ is not contained in $G^{\omega}$ let $K$ be the group generated by $G$ and $Z$. $G^{\omega}$ is normal in $K$ since

$$
\left[G^{\omega}, G\right] \subseteq G^{\omega} \text { and }\left[G^{\omega}, Z\right]=E \subseteq G^{\omega} .
$$

It follows that $Z$ is normal in $K$ and hence $K=Z G$. But $G$ is subinvariant in $A$, and hence in $K$. That is, $G$ is a proper normal subgroup of $G_{1}, G_{1}$ contained in $K$. Pick $g_{1}$ in $G_{1}$ but not in $G$. Since $K=Z G, g_{1}=g z$ where $g$ is in $G$, and $z$ in $Z$. Furthermore $z=g^{-1} g_{1}$ is in $G_{1}$ and not in $G$. Now $G$ and $z$ generate a group $L=G(z)$ since $G$ is normal in $G_{1}$. Also $L^{\omega}=G^{\omega}$; for

$$
L / G^{\omega}=G / G^{\omega} \times(z) G^{\omega} / G^{\omega}
$$

and hence is nilpotent.

Now since $z \notin G^{\omega}=L^{\omega}$ it follows by Lemma 2 that $L$ has a non-trivial center; but this is a contradiction of the fact that $G$ has centralizer $E$ in $A$. This completes the proof of the Theorem.

The counter-example mentioned earlier is as follows. Let $H$ be the non-Abelian group of order 27 all of whose elements are of order 3 ; and let $a$ and $b$ be generators of $H$. Let $\sigma$ and $\tau$ be automorphisms of $H$ defined by $a^{\sigma}=a^{2}, b^{\sigma}=b$; and $a^{\tau}=a, b^{\tau}=b^{2}$. Let $B$ be the holomorph of $H$ with $\sigma$ and $\tau$ and let $G$ be the subgroup of $B$ containing $\sigma$, $a$, and $[a, b]$. Then $G$ is invariant in the subgroup containing $G$ and $b$ which subgroup in turn is invariant in $B$; and it is easy to check that $G^{\omega}$ is the group generated by $a$ and $[a, b]$.

Now let $\rho$ be the automorphism of order 2 of $B$ defined by $b^{\rho}=a$, $a^{\rho}=b, \sigma^{\rho}=\tau, \tau^{\rho}=\sigma$, and let $\mathrm{A}$ be the holomorph of $B$ and $\rho$. Then $G$ is subinvariant in $A$; the centralizer of $G$ in $A$ is the identity, but clearly $G^{\omega}$ is not normal in $A$ since $a^{\rho}=b$.

Omitting the hypothesis of solvability Professor Zassenhaus kindly furnished me with a similar example; in fact, an example of a group $G=G^{2}$ with trivial center such that the group of automorphisms of $G$ is not complete. 


\section{REFERENCES}

1. Eugene Schenkman, A theory of subinvariant Lie algebras, Amer. J. Math., 73 (1951), 453-474.

2. H. Wielandt, Eine Verallgemeinerung der invarianten Untergruppen, Math. Zeitschr., 45 (1939), 209-244.

3. H. Zassenhaus, The Theory of Groups, translated by Saul Kravetz, Chelsea Publishing Company (New York, 1949).

LOUISIANA STATE UNIVERSITY AND

Institute FOR ADVANCED STUdy 
.././. ./FrontMatter/paper .pdf 


\section{Pacific Journal of Mathematics}

Nesmith Cornett Ankeny and Theodore Joseph Rivlin, On a theorem of S.

Bernstei........................................ 849

Louis Auslander, The use of forms in variational calculation .......... 853

Paul Civin, Abstract Riemann sum . .......................... 861

Paul Civin, Some ergodic theorems involving two operator ............ 869

Eckford Cohen, The number of solutions of certain cubic congruence .... . 877

Richard M. Cohn, Specializations over difference field .............. 887

Jean Dieudonné, Pseudo-discriminant and Dickson invarian . . ......... 907

Ky Fan, A comparison theorem for eigenvalues of normal matrice ........ 911

Richard P. Gosselin, On the convergence behaviour of trigonometric interpolating polynomial ........................... 915

Peter K. Henrici, On generating functions of the Jacobi polynomial . . . . . . . 923

Meyer Jerison, An algebra associated with a compact grou ............ 933

Wilhelm Magnus, Infinite determinants associated with Hill's equatio . . . . . 941

G. Power and D. L. Scott-Hutton, The slow steady motion of liquid past a semi-elliptical bos................................. 953

Lyle E. Pursell, An algebraic characterization of fixed ideals in certain function ring .................................... 963

C. T. Rajagopal, Additional note on some Tauberian theorems of O. Szás . . 971 Louis Baker Rall, Error bounds for iterative solutions of Fredholm integral

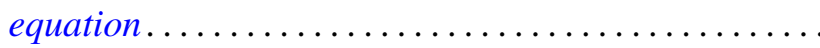

Shigeo Sasaki and Kentaro Yano, Pseudo-analytic vectors on

pseudo-Kählerian manifold ......................

Eugene Schenkman, On the tower theorem for finite group

P. Stein and John E. L. Peck, On the numerical solution of Poisson's equation over a rectangl ........................

Morgan Ward, The mappings of the positive integers into themselves which preserve divisio .

Seth Warner, Weak locally multiplicatively-convex algebra 1025

Louis Weisner, Group-theoretic origin of certain generating function .... 